\title{
IRREGULARITIES OF DISTRIBUTION. II
}

\author{
BY \\ WOLFGANG M. SCHMIDT $\left({ }^{1}\right)$
}

1. Introduction. Let $\boldsymbol{p}_{1}, \ldots, \boldsymbol{p}_{N}$ be points (not necessarily distinct) in the unit cube $0 \leqq x_{1}<1, \ldots, 0 \leqq x_{n}<1$ of Euclidean $E^{n}$ where $n>1$. Further let $\boldsymbol{P}$ be the set of points $p_{i}+\boldsymbol{g}$ where $p_{i}$ is one of the given points and $\boldsymbol{g}$ is an integer point. Given a bounded and Jordan-measurable set $A$ of measure $\mu(A)$, write $\nu(A)$ for the number (counted with multiplicities) of points of $\boldsymbol{P}$ in $\boldsymbol{A}$, and put

$$
\Delta(A)=|N \mu(A)-v(A)| .
$$

Given an arbitrary point $\boldsymbol{u}$ with coordinates $\left(u_{1}, \ldots, u_{n}\right)$, write $\boldsymbol{B}(\boldsymbol{u})$ for the box consisting of the points $\boldsymbol{x}=\left(x_{1}, \ldots, x_{n}\right)$ having $0 \leqq x_{j}<\left|u_{j}\right|(j=1, \ldots, n)$. By a theorem of Roth [3], there exist boxes $\boldsymbol{B}(\boldsymbol{u})$ contained in the unit cube having

$$
\Delta(\boldsymbol{B}) \geqq c_{1}(n)(\log N)^{(n-1) / 2} .
$$

(The constant $c_{1}$ and other constants $c_{2}, c_{3}, \ldots$ introduced below are positive and independent of $N$.) It is not known whether this estimate is best possible, but it can be shown that the exponent of the logarithm in (2) cannot be replaced by numbers larger than a certain constant $c_{2}(n)$. (One may take $c_{2}(2)=1$ by [3, inequality (3)], and when $n>2$ one may take $c_{2}(n)=n$ by [4, Theorem 3], since the estimate given there is easily checked to be independent of the box considered.)

Little could be gained by allowing translations of the boxes $\boldsymbol{B}(\boldsymbol{u})$. Namely, if $\boldsymbol{u}, \boldsymbol{v}$ are any points, let $\boldsymbol{B}(\boldsymbol{u} ; \boldsymbol{v})$ be the box consisting of the points $\boldsymbol{x}+\boldsymbol{v}$ where $\boldsymbol{x} \in \boldsymbol{B}(\boldsymbol{u})$. Now suppose that both $\boldsymbol{u}, \boldsymbol{v}$ have positive coordinates, and set $\boldsymbol{B}=\boldsymbol{B}(\boldsymbol{u} ; \boldsymbol{v})$. Then at least one of the $2^{n}$ boxes $\boldsymbol{B}^{\prime}=\boldsymbol{B}(\boldsymbol{w})$ where each coordinate $w_{j}$ of $\boldsymbol{w}$ is either $v_{j}$ or $u_{j}+v_{j}$, satisfies $\Delta\left(\boldsymbol{B}^{\prime}\right) \geqq 2^{-n} \Delta(\boldsymbol{B})$.

The situation is rather different if we allow rotations. If $\tau$ is an orthogonal transformation of $E^{n}$, write $\boldsymbol{B}(\boldsymbol{u} ; \boldsymbol{v} ; \tau)$ for the box consisting of the points $\tau \boldsymbol{x}+\boldsymbol{v}$ where $\boldsymbol{x} \in \boldsymbol{B}(\boldsymbol{u})$. The diameter of this box is $|\boldsymbol{u}|=\left(u_{1}^{2}+\cdots+u_{1}^{2}\right)^{1 / 2}$.

Theorem 1. Suppose $n=2$ and $\delta$ is real and satisfies $N \delta^{2}>1$. Then there is a rectangle $\boldsymbol{B}=\boldsymbol{B}(\boldsymbol{u} ; \boldsymbol{v} ; \boldsymbol{\tau})$ of diameter $\delta$ with

$$
\Delta(\boldsymbol{B}) \geqq c_{3}\left(\log \left(N \delta^{2}\right)\right)^{1 / 2} .
$$

TheOREM 2. Suppose $n>2$ and $\delta, \varepsilon$ are real and have $N \delta^{n}>\varepsilon>0$. There is a box $\boldsymbol{B}=\boldsymbol{B}(\boldsymbol{u} ; \boldsymbol{v} ; \tau)$ of diameter $\delta$ satisfying

$$
\Delta(\boldsymbol{B}) \geqq c_{4}(\varepsilon, n)\left(N \delta^{n}\right)^{1 / 6} .
$$

Received by the editors November 8, 1967.

(1) Written with partial support from the National Science Foundation Grant No. NSFGP-6515. 
Note that the lower bound here involves a power of $N$ rather than a power of $\log N$. Theorem 2 will turn out to be an easy consequence of its special case $n=3$. If we were able to give a direct proof of a theorem of this type when $n>3$, then the exponent $1 / 6$ could probably be replaced by an exponent depending on $n$ and increasing with $n$. However, it would be impossible to replace $1 / 6$ by a number greater than $1 / 2$ by the law of the iterated logarithm (which holds for almost all sequences in the sense of the infinite product measure $)\left({ }^{2}\right)$.

Theorems 1, 2 will be derived from certain integral inequalities. In this respect our approach is like Roth's [3], but our proof of the integral inequalities is quite different from Roth's.

2. Integral inequalities. Write $U$ for the unit cube of $E^{n}$. Roth proved the inequality

$$
\int_{U} \Delta(\boldsymbol{B}(\boldsymbol{u}))^{2} d \boldsymbol{u} \geqq c_{5}(n)(\log N)^{n-1},
$$

which implies the existence of a box $\boldsymbol{B}(\boldsymbol{u})$ satisfying (2).

Let $S$ be the unit sphere consisting of points $\sigma$ with $|\sigma|=1$, and let $d \sigma$ be the $\left((n-1)\right.$-dimensional) volume element on $S$, normalized such that $\int_{S} d \sigma=1$. Any box $\boldsymbol{B}(\boldsymbol{u} ; \boldsymbol{v} ; \tau)$ of diameter $\delta$ has $\boldsymbol{u}$ of the type $\boldsymbol{u}=\delta \boldsymbol{\sigma}$ with $\boldsymbol{\sigma} \in S$.

Let $T$ be the group of proper orthogonal transformations and $d \tau$ the volume element of the invariant measure on $T$, normalized such that $\int_{T} d \tau=1$.

THEOREM $1^{\prime}$. Suppose $n=2$ and $\delta>0$. Then

$$
\int_{T} d \tau \int_{U} d \boldsymbol{u} \int_{S} d \boldsymbol{\sigma} \Delta(\boldsymbol{B}(\delta \boldsymbol{\sigma} ; \boldsymbol{u} ; \tau))^{2} \geqq c_{6} \log \left(N \delta^{2}\right) .
$$

Theorem 1 follows immediately.

TheOrem $2^{\prime}$. Suppose $n=3$ and $N \delta^{3}>\varepsilon>0$. Then

$$
\int_{T} \dot{a}: \int_{U} d \boldsymbol{u} \int_{S} d \boldsymbol{\sigma} \Delta(\boldsymbol{B}(\delta \boldsymbol{\sigma} ; \boldsymbol{u} ; \tau))^{2} \geqq c_{7}(\varepsilon)\left(N \delta^{3}\right)^{1 / 3}
$$

I am unable to generalize this theorem to higher dimensions. Theorem $2^{\prime}$ implies the case $n=3$ of Theorem 2. The general case of Theorem 2 may be proved by induction on $n$ as follows.

Suppose $n>3$ and Theorem 2 has been shown for $n-1$. As is easily seen, there is a real number $x$ with

$$
N(x)=\nu(\boldsymbol{B}((\delta / \sqrt{ } 2,1, \ldots, 1) ;(x, 0, \ldots, 0))) \geqq N \delta / \sqrt{ } 2 .
$$

$\left({ }^{2}\right)$ Added in proof. To prove this probabilistic result one has to modify Satz 2 in [W. Philipp, Das Gesetz vom iterierten Logarithmus mit Anw. auf die Zahlentheorie, Math. Ann. (to appear)]. In the fourth paper of this series we shall use the integral equations established in this paper to prove stronger versions of Theorems 1, 2 . 
We shall distinguish two cases (a) and (b).

(a) $(N(x)-N \delta / \sqrt{ } 2) \delta^{n-1} \geqq 2^{-n} c_{4}\left(\varepsilon 2^{-n}, n-1\right)\left(N \delta^{n}\right)^{1 / 6}$.

In this case we have

$$
\begin{aligned}
\int_{0}^{1} & \cdots \int_{0}^{1} \Delta\left(\boldsymbol{B}\left(\left(\delta 2^{-1 / 2}, \delta(2 n-2)^{-1 / 2}, \ldots, \delta(2 n-2)^{-1 / 2}\right),\left(x, x_{2}, \ldots, x_{n}\right)\right)\right) d x_{2} \cdots d x_{n} \\
\geqq & \int_{0}^{1} \ldots \int_{0}^{1} \nu\left(\boldsymbol{B}\left(\left(\delta 2^{-1 / 2}, \delta(2 n-2)^{-1 / 2}, \ldots, \delta(2 n-2)^{-1 / 2}\right),\left(x, x_{2}, \ldots, x_{n}\right)\right)\right) d x_{2} \cdots d x_{n} \\
& \quad-N \delta^{n} 2^{-1 / 2}(2 n-2)^{-(n-1) / 2} \\
= & (N(x)-N \delta / \sqrt{ } 2) \delta^{n-1}(2 n-2)^{-(n-1) / 2} \\
\geqq & (4 n)^{-n} c_{4}\left(\varepsilon 2^{-n}, n-1\right)\left(N \delta^{n}\right)^{1 / 6},
\end{aligned}
$$

and there is a box $\boldsymbol{B}$ of diameter $\delta$ having

$$
\Delta(B) \geqq(4 n)^{-n} c_{4}\left(\varepsilon 2^{-n}, n-1\right)\left(N \delta^{n}\right)^{1 / 6} .
$$

(b) $(N(x)-N \delta / \sqrt{ } 2) \delta^{n-1}<2^{-n} c_{4}\left(\varepsilon 2^{-n}, n-1\right)\left(N \delta^{n}\right)^{1 / 6}$.

Let $\boldsymbol{p}_{1}, \ldots, \boldsymbol{p}_{N(x)}$, say, be the points counted by $N(x)$. Further let $\boldsymbol{p}_{1}^{\prime}, \ldots, \boldsymbol{p}_{N(x)}^{\prime}$ be their orthogonal projections on the subspace $x_{1}=0$. We then have

$$
N(x)(\delta / \sqrt{ } 2)^{n-1} \geqq N \delta^{n} 2^{-n / 2}>\varepsilon 2^{-n},
$$

and by our inductive assumption there is an $(n-1)$-dimensional box $\boldsymbol{B}^{\prime}$ in this subspace of diameter $\delta / \sqrt{ } 2$ satisfying

$$
\Delta\left(\boldsymbol{B}^{\prime}\right)=\left|N(x) \mu\left(\boldsymbol{B}^{\prime}\right)-\nu\left(\boldsymbol{B}^{\prime}\right)\right| \geqq c_{4}\left(\varepsilon 2^{-n}, n-1\right)\left(N(x)(\delta / \sqrt{ } 2)^{n-1}\right)^{1 / 6} .
$$

Let $\boldsymbol{B}$ be the box in $E^{n}$ consisting of the points $\boldsymbol{x}=\left(x_{1}, x_{2}, \ldots, x_{n}\right)$ with $x \leqq x_{1}$ $<x+\delta / \sqrt{ } 2$ and $\left(x_{2}, \ldots, x_{n}\right)$ in $\boldsymbol{B}^{\prime}$. Then

$$
\begin{aligned}
\Delta(\boldsymbol{B}) & =|N \mu(\boldsymbol{B})-\nu(\boldsymbol{B})| \\
& =\left|N \mu\left(\boldsymbol{B}^{\prime}\right) \delta / \sqrt{ } 2-\nu\left(\boldsymbol{B}^{\prime}\right)\right| \\
& \geqq\left|N(x) \mu\left(\boldsymbol{B}^{\prime}\right)-\nu\left(\boldsymbol{B}^{\prime}\right)\right|-|N \delta / \sqrt{ } 2-N(x)| \mu\left(\boldsymbol{B}^{\prime}\right) \\
& \geqq c_{4}\left(\varepsilon 2^{-n}, n-1\right)\left(N(x)(\delta / \sqrt{ } 2)^{n-1}\right)^{1 / 6}-2^{-n} c_{4}\left(\varepsilon 2^{-n}, n-1\right)\left(N \delta^{n}\right)^{1 / 6} \\
& \geqq(4 n)^{-n} c_{4}\left(\varepsilon 2^{-n}, n-1\right)\left(N \delta^{n}\right)^{1 / 6} .
\end{aligned}
$$

Here we used the inequalities $\mu\left(\boldsymbol{B}^{\prime}\right)<\delta^{n-1}$ and $N(x) \geqq N \delta / \sqrt{ } 2$.

3. An integral equation. Now suppose that

$$
0<|u|<1 / 4 \text {. }
$$

Put

and further put

$$
\begin{aligned}
f_{B}(\boldsymbol{u} ; \boldsymbol{v} ; \boldsymbol{\tau} \mid \boldsymbol{x}) & =1, \quad \text { if } \boldsymbol{x} \in \boldsymbol{B}(\boldsymbol{u} ; \boldsymbol{v} ; \tau), \\
& =0, \quad \text { otherwise }
\end{aligned}
$$

$$
g_{B}(\boldsymbol{u} ; \boldsymbol{v} ; \boldsymbol{\tau} \mid \boldsymbol{x})=\sum_{\boldsymbol{g}} f_{B}(\boldsymbol{u} ; \boldsymbol{v} ; \boldsymbol{\tau} \mid \boldsymbol{x}+\boldsymbol{g}),
$$


where the summation is over all integer points $g$. Also write

$$
h_{B}(\boldsymbol{u} ; \boldsymbol{x}, \boldsymbol{y})=\int_{T} d \tau \int_{U} d \boldsymbol{v} g_{B}(\boldsymbol{u} ; \boldsymbol{v} ; \boldsymbol{\tau} \mid \boldsymbol{x}) g_{B}(\boldsymbol{u} ; \boldsymbol{v} ; \boldsymbol{\tau} \mid \boldsymbol{y}) .
$$

Put $\omega(\boldsymbol{x}, \boldsymbol{y})$ for the minimum of the distances $|(\boldsymbol{x}+\boldsymbol{g})-\boldsymbol{y}|$, taken over all integer points $\boldsymbol{g}$. Let $\boldsymbol{x}_{1}$ be a point $\boldsymbol{x}+\boldsymbol{g}$ with $\omega(\boldsymbol{x}, \boldsymbol{y})=\left|\boldsymbol{x}_{1}-\boldsymbol{y}\right|$.

Since $g_{B}(\boldsymbol{u} ; \boldsymbol{v} ; \tau \mid \boldsymbol{x})$ is periodic in $\boldsymbol{v}$ and in $\boldsymbol{x}$, we have

$$
\begin{aligned}
h_{B}(\boldsymbol{u} ; \boldsymbol{x}, \boldsymbol{y}) & =h_{B}\left(\boldsymbol{u} ; \boldsymbol{x}_{1}, \boldsymbol{y}\right) \\
& =\int_{T} d \tau \int_{U} d \boldsymbol{v} g_{B}\left(\boldsymbol{u} ; \boldsymbol{v}-\boldsymbol{y} ; \tau \mid \boldsymbol{x}_{1}-\boldsymbol{y}\right) g_{B}(\boldsymbol{u} ; \boldsymbol{v}-\boldsymbol{y} ; \tau \mid \mathbf{0}) \\
& =h_{B}\left(\boldsymbol{u} ; \boldsymbol{x}_{1}-\boldsymbol{y}, \mathbf{0}\right) .
\end{aligned}
$$

The box $\boldsymbol{B}(\boldsymbol{u})$ has diameter $|\boldsymbol{u}|$, and therefore $h_{B}(\boldsymbol{u} ; \boldsymbol{z}, \mathbf{0})=0$ unless $|\boldsymbol{z}| \leqq|\boldsymbol{u}|<1 / 4$. It therefore will suffice to investigate the function $h_{B}(\boldsymbol{u} ; \boldsymbol{z}, \mathbf{0})$ with $\boldsymbol{z}$ satisfying

$$
|z| \leqq|u|<1 / 4
$$

In the definition (9) of $h_{B}$ one may replace the cube $U$ by the cube $U^{\prime}$ consisting of points $\boldsymbol{x}$ with $-1 / 2 \leqq x_{i}<1 / 2(i=1, \ldots, n)$. But if $\boldsymbol{u}$ satisfies (8) and if $\boldsymbol{v} \in U^{\prime}$, then $g_{B}(\boldsymbol{u} ; \boldsymbol{v} ; \boldsymbol{\tau} \mid \boldsymbol{0})$ is zero unless $|\boldsymbol{v}| \leqq|\boldsymbol{u}|$, and then by (10) the functions $f_{B}(\boldsymbol{u} ; \boldsymbol{v} ; \boldsymbol{\tau} \mid \boldsymbol{g})$ and $f_{B}(\boldsymbol{u} ; \boldsymbol{v} ; \boldsymbol{\tau} \mid \boldsymbol{z}+\boldsymbol{g})$ are zero unless $\boldsymbol{g}=\mathbf{0}$. Hence

$$
\begin{aligned}
h_{B}(\boldsymbol{u} ; \boldsymbol{z}, \mathbf{0}) & =\int_{T} d \tau \int_{|v| \leqq|u|} d v f_{B}(\boldsymbol{u} ; \boldsymbol{v} ; \tau \mid \boldsymbol{z}) f_{B}(\boldsymbol{u} ; \boldsymbol{v} ; \tau \mid \mathbf{0}) \\
& =\int_{T} d \tau \int_{E^{n}} d v f_{B}\left(\boldsymbol{u} ; \tau^{-1} v ; \iota \mid \tau^{-1} z\right) f_{B}\left(\boldsymbol{u} ; \tau^{-1} v ; \iota \mid 0\right)
\end{aligned}
$$

where $\iota$ is the identity map. We obtain

$$
\begin{aligned}
h_{B}(\boldsymbol{u} ; \boldsymbol{z}, \mathbf{0}) & =\int_{\boldsymbol{T}} d \tau \int_{E^{n}} d \boldsymbol{v} f_{B}(\boldsymbol{u} ; \boldsymbol{v} ; \iota \mid \tau z) f_{B}(\boldsymbol{u} ; \boldsymbol{v} ; \iota \mid \mathbf{0}) \\
& =\int_{S} d \boldsymbol{\sigma} \int_{E^{n}} d \boldsymbol{v} f_{B}(\boldsymbol{u} ; \boldsymbol{v} ; \iota|| \boldsymbol{z} \mid \boldsymbol{\sigma}) f_{B}(\boldsymbol{u} ; \boldsymbol{v} ; \iota \mid \mathbf{0}) .
\end{aligned}
$$

Therefore $h_{B}(\boldsymbol{u} ; \boldsymbol{z}, \boldsymbol{0})$ as a function of $z$ depends only on $|\boldsymbol{z}|$, and $h_{B}(\boldsymbol{u} ; \boldsymbol{x}, \boldsymbol{y})$ as a function of $\boldsymbol{x}, \boldsymbol{y}$ depends only on $\omega(\boldsymbol{x}, \boldsymbol{y})$ :

$$
h_{B}(\boldsymbol{u} ; \boldsymbol{x} ; \boldsymbol{y})=k_{B}(\boldsymbol{u} ; \omega(\boldsymbol{x}, \boldsymbol{y})) .
$$

Note that $0 \leqq \omega(x, y) \leqq \sqrt{ }(n) / 2$, and hence (12) defines $k_{B}(\boldsymbol{u} ; \omega)$ only if $\boldsymbol{u}$ satisfies (8) and if $0 \leqq \omega \leqq \sqrt{ }(n) / 2$. We now extend this definition by setting $k_{B}(\boldsymbol{u} ; \omega)=0$ if $\omega>\sqrt{ }(n) / 2$. We have

$$
\begin{aligned}
\int_{U} \int_{U} k_{B}(\boldsymbol{u} ; \omega & (\boldsymbol{x}, \boldsymbol{y})) d \boldsymbol{x} d \boldsymbol{y} \\
& =\int_{T} d \tau \int_{U} d v \iint_{U U} d \boldsymbol{x} d \boldsymbol{y} g_{B}(\boldsymbol{u} ; \boldsymbol{v} ; \tau \mid \boldsymbol{x}) g_{B}(\boldsymbol{u} ; \boldsymbol{v} ; \tau \mid \boldsymbol{y}) \\
& =\mu(\boldsymbol{B}(\boldsymbol{u}))^{2} .
\end{aligned}
$$


Now suppose the points $\boldsymbol{p}_{1}, \ldots, \boldsymbol{p}_{N}$ and the set $\boldsymbol{P}$ are as above. Then

$$
\begin{aligned}
\int_{T} d \tau \int_{U} d v & \Delta(\boldsymbol{B}(\boldsymbol{u} ; \boldsymbol{v} ; \tau))^{2} \\
= & N^{2} \mu(\boldsymbol{B}(\boldsymbol{u}))^{2}-2 N \mu(\boldsymbol{B}(\boldsymbol{u})) \int_{T} d \tau \int_{U} d v v(\boldsymbol{B}(\boldsymbol{u} ; \boldsymbol{v} ; \tau)) \\
& +\int_{T} d \tau \int_{U} d \boldsymbol{v} \nu^{2}(\boldsymbol{B}(\boldsymbol{u} ; \boldsymbol{v} ; \tau))^{2} \\
= & -N^{2} \mu(\boldsymbol{B}(\boldsymbol{u}))^{2}+\sum_{i, j=1}^{N} \int_{T} d \tau \int_{U} d v g_{B}\left(\boldsymbol{u} ; \boldsymbol{v} ; \tau \mid \boldsymbol{p}_{i}\right) g_{B}\left(\boldsymbol{u} ; \boldsymbol{v} ; \tau \mid \boldsymbol{p}_{j}\right) \\
= & \sum_{i, j=1}^{N}\left(h_{B}\left(\boldsymbol{u} ; \boldsymbol{p}_{i}, \boldsymbol{p}_{j}\right)-\mu(\boldsymbol{B}(\boldsymbol{u}))^{2}\right) \\
= & \sum_{i, j=1}^{N}\left(k_{B}\left(\boldsymbol{u} ; \omega\left(\boldsymbol{p}_{i}, \boldsymbol{p}_{j}\right)\right)-\int_{U} \int_{U} k_{B}(\boldsymbol{u} ; \omega(\boldsymbol{x}, \boldsymbol{y})) d \boldsymbol{x} d \boldsymbol{y}\right) .
\end{aligned}
$$

Let $\boldsymbol{C}(\rho)$ be the ball consisting of the points $\boldsymbol{x}$ with $|\boldsymbol{x}| \leqq \rho$. If $\boldsymbol{v}$ is a point and $\tau$ an orthogonal transformation of $E^{n}$, let $C(\rho ; v ; \tau)$ be the ball consisting of the points $\tau \boldsymbol{x}+\boldsymbol{v}$ where $\boldsymbol{x} \in \boldsymbol{C}(\rho)$. (The transformation $\tau$ has no effect here, but is retained to stress the analogy with $\boldsymbol{B}(\boldsymbol{u} ; \boldsymbol{v} ; \tau)$.)

Now assume that

$$
0<\rho<1 / 8
$$

and put

$$
\begin{aligned}
f_{C}(\rho ; \boldsymbol{v} ; \tau \mid \boldsymbol{x}) & =1 & & \text { if } \boldsymbol{x} \in \boldsymbol{C}(\rho ; \boldsymbol{v} ; \tau), \\
& =0 & & \text { otherwise, }
\end{aligned}
$$

and define $g_{C}(\rho ; \boldsymbol{v} ; \boldsymbol{\tau} \mid \boldsymbol{x}), h_{C}(\rho ; \boldsymbol{x}, \boldsymbol{y}), k_{C}(\rho ; \omega)$ in the obvious way. Then

$$
\begin{aligned}
& \int_{T} d \tau \int_{U} d v \Delta(\boldsymbol{C}(\rho ; \boldsymbol{v} ; \tau))^{2} \\
&=\sum_{i, j=1}^{N}\left(k_{C}\left(\rho ; \omega\left(\boldsymbol{p}_{i}, \boldsymbol{p}_{j}\right)\right)-\int_{U} \int_{U} k_{C}(\rho ; \omega(\boldsymbol{x}, \boldsymbol{y})) d \boldsymbol{x} d \boldsymbol{y}\right) .
\end{aligned}
$$

Lemma 1. Suppose $0<\delta<1 / 4$ and suppose the function $f(x)$ is nonnegative and continuous in $0<x \leqq 1 / 2$ and satisfies the integral equation

$$
\int_{0}^{1 / 2} f(\rho) k_{C}(\delta \rho ; \omega) d \rho=\int_{S} k_{B}(\delta \sigma ; \omega) d \sigma \quad(\omega \geqq 0) .
$$

Then

(18) $\int_{T} d \tau \int_{U} d v \int_{0}^{1 / 2} d \rho f(\rho) \Delta(C(\delta \rho ; v ; \tau))^{2}=\int_{T} d \tau \int_{U} d v \int_{S} d \boldsymbol{\sigma} \Delta(\boldsymbol{B}(\delta \boldsymbol{\sigma} ; \boldsymbol{v} ; \tau))^{2}$.

REMARKS. The substitution $\delta \rightarrow c \delta, \omega \rightarrow c \omega$ has the effect of multiplying both sides of (17) by $c^{n}$, since both $k_{B}$ and $k_{C}$ are homogeneous of degree $n$. Hence if $f$ solves the equation for some particular $\delta$, then it solves it for every $\delta$ in $0<\delta<1 / 4$. 
(Note that $k_{B}(\delta \sigma ; \omega)$ is only defined in $0<\delta<1 / 4$.) Moreover, both sides of (18) remain invariant if $\boldsymbol{P}$ is replaced by the contracted set $(1 / m) \boldsymbol{P}, N$ by $m^{n} N$, and $\delta$ by $\delta / m$, where $m$ is a positive integer. Hence if (18) holds for every $\delta$ in $0<\delta<1 / 4$, then it holds for every $\delta>0$.

Proof. The relation (18) follows from (14), (16) and (17).

4. Applying the integral equation. It is perhaps time to explain how our machinery will be used to prove the desired theorems.

It will suffice to find a solution $f(x)$ of (17) and then to give a lower bound for the left hand side of (18). Surprisingly, the trivial estimate

$$
\Delta(\boldsymbol{C}(\rho ; \boldsymbol{v} ; \tau)) \geqq\|N \mu(\boldsymbol{C}(\rho))\|
$$

where \|\| denotes the distance from the nearest integer, will be all we need to know about $\Delta(\boldsymbol{C}(\rho ; \boldsymbol{v} ; \tau))$.

When $n=2$ we shall find a positive solution $f(x)$ of (17) which satisfies

$$
f(x) \geqq c_{8} / x
$$

in some interval $0<x<c_{9}$. By (18), (19), and (20),

$$
\int_{T} d \tau \int_{U} d v \int_{S} d \sigma \Delta(B(\delta \sigma ; v ; \tau))^{2} \geqq c_{8} \int_{0}^{c_{9}} \rho^{-1}\left\|N \rho^{2} \delta^{2} \pi\right\|^{2} d \rho .
$$

Putting $x=N \rho^{2} \delta^{2} \pi$ we obtain

$$
\frac{1}{2} c_{8} \int_{0}^{c_{10} N \delta^{2}} x^{-1}\|x\|^{2} d x \geqq c_{6} \log \left(N \delta^{2}\right) .
$$

When $n=3$ we shall find a positive solution of (17) with

$$
f(x) \geqq c_{11} / x^{2}
$$

in some interval $0<x<c_{12}$. The left hand side and therefore also the right hand side of (18) exceeds

$$
c_{11} \int_{0}^{c_{12}} \rho^{-2}\left\|N \rho^{3} \delta^{3} 4 \pi / 3\right\|^{2} d \rho
$$

Putting $y=N \rho^{3} \delta^{3} 4 \pi / 3$ we obtain the lower bound

$$
c_{13} N^{1 / 3} \delta \int_{0}^{c_{14} N \delta^{3}} y^{-4 / 3}\|y\|^{2} d y
$$

which is at least $c_{7}(\varepsilon)\left(N \delta^{3}\right)^{1 / 3}$ provided $N \delta^{3}>\varepsilon$.

In principle there is no reason why our method could not be used in many similar situations, but in general it is hard to show that the corresponding integral equation has a suitable solution.

5. A lemma in real analysis. We shall have occasion to use the following lemma. 
Lemma 2. Suppose $u(x), v(x)$ are nondecreasing functions defined in $a<x<b$ which satisfy $u(x)<v(x)$ and have continuous derivatives. Let $A$ be the set of points $(x, y)$ with $a<x<b, u(x) \leqq y \leqq v(x)$, and $A^{\prime}$ the set of points with $a<x<b, u(x)$ $<y \leqq v(x)$.

Further let $f(x, y)$ be a function which is defined and continuous in $A$ and has a continuous partial derivative $\partial f / \partial x$ in $A^{\prime}$ such that

$$
|\partial f| \partial x \mid \ll(y-u(x))^{-1 / 2}
$$

if $(x, y)$ is in $A^{\prime}$ and $x$ in a fixed closed subinterval of $a<x<b$.

Then $F(x)=\int_{u(x)}^{v(x)} f(x, y) d y$ has the derivative

$$
F^{\prime}(x)=\int_{u(x)}^{v(x)} \frac{\partial f}{\partial x}(x, y) d y+v^{\prime}(x) f(x, v(x))-u^{\prime}(x) f(x, u(x))
$$

in $a<x<b$.

Proof. This lemma may be easily derived from [1, Theorem XI], say. It probably can also be found in other places of the literature. A direct proof is as follows.

Let $x$ be in $a<x<b$, and let $h$ be positive and so small that $x+h<b$ and $u(x+h)$ $<v(x)$. Then

$$
\begin{aligned}
h^{-1}(F(x+h)-F(x))= & h^{-1} \int_{u(x+h)}^{v(x)}(f(x+h, y)-f(x, y)) d y \\
& \quad+h^{-1} \int_{v(x)}^{v(x+h)} f(x+h, y) d y-h^{-1} \int_{u(x)}^{u(x+h)} f(x, y) d y \\
& =I_{1}+I_{2}-I_{3},
\end{aligned}
$$

say. One has

$$
I_{1}=\int_{u(x+h)}^{v(x)} \frac{\partial f}{\partial x}(x+\tau(h, y), y) d y,
$$

where $0<\tau(h, y)<h$. For any $\varepsilon>0$ with $u(x+h)+\varepsilon<v(x)$ this integral may be split into

$$
\int_{u(x+h)}^{u(x+h)+\varepsilon}+\int_{u(x+h)+\varepsilon}^{v(x)}
$$

The first integral here is $O\left(\varepsilon^{1 / 2}\right)$ by (22). On the other hand, by the continuity of $\partial f / \partial x$ in $A^{\prime}$, the second integral tends to

$$
\int_{u(x)+\varepsilon}^{v(x)} \frac{\partial f}{\partial x}(x, y) d y
$$

when $\varepsilon>0$ is fixed and $h \rightarrow 0^{+}$. Again by (22), the integral (24) differs from

$$
\int_{u(x)}^{v(x)} \frac{\partial f}{\partial x}(x, y) d y
$$

by at most $O\left(\varepsilon^{1 / 2}\right)$. Since $\varepsilon>0$ was arbitrarily small, we see that $I_{1}$ tends to (25) as $h \rightarrow 0^{+}$. 
Next,

$$
I_{2}=h^{-1}(v(x+h)-v(x)) f(x+h, \rho(h))=v^{\prime}(x+\sigma(h)) f(x+h, \rho(h))
$$

where $v(x) \leqq \rho(h) \leqq v(x+h)$ and $0<\sigma(h)<h$. Hence $I_{2}$ tends to $v^{\prime}(x) f(x, v(x))$ as $h \rightarrow 0^{+}$. The integral $I_{3}$ is treated similarly.

The situation is analogous when $h$ is negative.

6. Transformation of the integral equation. Suppose that $0<|\boldsymbol{u}|<1 / 4$, i.e: that (8) holds. Then

$$
k_{B}(u ; \omega)=\int_{S} d \boldsymbol{\sigma} \int_{E^{n}} d v f_{B}(u ; v ; \iota \mid \omega \sigma) f_{B}(u ; v ; \iota \mid 0) .
$$

This is true by (11) and (12) if $\omega \leqq \sqrt{ }(n) / 2$, and it is true if $\omega>\sqrt{ }(n) / 2>|u|$, since then both sides of (26) are zero. In the range $0<|\boldsymbol{u}|<1 / 4$, the function $k_{B}(\boldsymbol{u} ; \omega)$ is homogeneous of degree $n$; that is, $k_{B}(t u ; t \omega)=t^{n} k_{B}(u ; \omega)$. Let $k_{B}^{*}(u ; \omega)$ be the unique function defined for $\boldsymbol{u} \neq 0$ and $\omega \geqq 0$ which is homogeneous of degree $n$ and coincides with $k_{B}(\boldsymbol{u} ; \omega)$ in $0<|\boldsymbol{u}|<1 / 4$. Then $k_{B}^{*}(\boldsymbol{u} ; \omega)$ is equal to the right hand side of (26). It is zero when $\omega>|\boldsymbol{u}|$.

Similarly, the function

$$
k_{C}^{*}(\rho ; \omega)=\int_{S} d \boldsymbol{\sigma} \int_{E^{n}} d v f_{C}(\rho ; v ; \iota \mid \omega \boldsymbol{\sigma}) f_{C}(\rho ; v ; \iota \mid \mathbf{0})
$$

is homogeneous and coincides with $k_{C}(\rho ; \omega)$ in $0<\rho<1 / 8$. It vanishes when $\omega>2 \rho$.

We may in (17) replace $k_{B}, k_{C}$ by $k_{B}^{*}, k_{C}^{*}$, respectively, and then set $\delta=1$. Then (17) becomes

$$
\int_{0}^{1 / 2} f(x) k_{C}^{*}(x ; y) d x=\int_{S} k_{B}^{*}(\sigma ; y) d \sigma \quad(0 \leqq y \leqq 1) .
$$

To solve this equation we need information about the function

$$
l(y)=\int_{S} k_{B}^{*}(\sigma ; y) d \sigma .
$$

Lemma 3. Suppose $n=2$. Then $l(y)$ and its derivatives $l^{\prime}(y), l^{\prime \prime}(y)$ are continuous in $0 \leqq y \leqq 1$, and in fact we have

$$
\begin{aligned}
& l(y)=2 \pi^{-2}\left(\left(1+2 y^{2}\right) \arccos y-3 y\left(1-y^{2}\right)^{1 / 2}\right), \\
& l^{\prime}(y)=8 \pi^{-2}\left(y \arccos y-\left(1-y^{2}\right)^{1 / 2}\right), \\
& l^{\prime \prime}(y)=8 \pi^{-2} \arccos y .
\end{aligned}
$$

Furthermore, in the interval $0 \leqq y<1$ we have

$$
l^{\prime \prime \prime}(y)=-8 \pi^{-2}\left(1-y^{2}\right)^{-1 / 2} .
$$

Lemma 4. Suppose $n=3$. Then

(i) $l(y)$ is continuous in $0 \leqq y \leqq 1$, and $l(1)=0$,

(ii) $l^{\prime}(y)$ is continuous in $0<y<1$, and $l(y) \rightarrow 0$ as $v \rightarrow 1$, 
(iii) $l^{\prime \prime}(y)$ is positive and continuous in $0<y<1$, and $l^{\prime \prime}(y) \rightarrow 0$ as $y \rightarrow 1$, and $l^{\prime \prime}(y) \rightarrow c_{15}>0$ when $y \rightarrow 0$,

(iv) $l^{\prime \prime \prime}(y)$ is negative and continuous in $0<y<1$, and $l^{\prime \prime \prime}(y) \rightarrow 0$ as $y \rightarrow 1$, and $\left|l^{\prime \prime \prime}(y)\right| \ll y^{-1 / 2}$ as $y \rightarrow 0$.

We postpone the proofs of these lemmas to the end of the paper. Assume from now on that

$$
n=2 \text { or } n=3 \text {. }
$$

The "kernel" $k_{C}^{*}(x, y)$ is equal to the volume of the intersection of two balls of radius $x$ whose centers have distance $y$. Hence

$$
k_{C}^{*}(x ; y)=c_{16}(n) \int_{y / 2}^{x}\left(x^{2}-t^{2}\right)^{(n-1) / 2} d t
$$

if $0 \leqq y \leqq 2 x$, and $k_{C}^{*}(x ; y)=0$ otherwise. In particular,

$$
k_{C}^{*}(x ; y) \ll x^{n} .
$$

In the interval $0 \leqq y<2 x$,

$$
\begin{aligned}
\frac{\partial}{\partial y} k_{C}^{*}(x ; y) & =-c_{17}(n)\left(x^{2}-y^{2} / 4\right)^{(n-1) / 2}, \\
\frac{\partial^{2}}{\partial y^{2}} k_{C}^{*}(x ; y) & =c_{18}(n) y\left(x^{2}-y^{2} / 4\right)^{(n-3) / 2} .
\end{aligned}
$$

Suppose a function $f(x)$ is continuous in $0<x \leqq 1 / 2$ and satisfies

$$
|f(x)| \ll x^{1-n}
$$

as $x \rightarrow 0$. Then by (30) the function $f(x) k_{C}^{*}(x ; y)$ tends to zero as $x \rightarrow 0$ and it can therefore be extended to a continuous function defined in $0 \leqq x \leqq 1 / 2,0 \leqq y \leqq 1$. Therefore the left hand side, and hence both sides of (28) are continuous functions of $y$, and it will suffice to verify (28) in $0<y<1$. The integral equation therefore may be rewritten as

$$
\int_{y / 2}^{1 / 2} f(x) k_{C}^{*}(x ; y) d x=l(y) \quad(0<y<1) .
$$

The kernel $k_{C}^{*}(x ; y)$ vanishes on the line $x=y / 2$, and hence the equation is a Volterra equation of the first kind [2, Chapter 1, paragraph 8].

Suppose (32) holds. Taking the derivative on both sides we obtain

$$
-c_{17}(n) \int_{y / 2}^{1 / 2} f(x)\left(x^{2}-y^{2} / 4\right)^{(n-1) / 2} d x=l^{\prime}(y) \quad(0<y<1) .
$$

Conversely, this equation implies (32), since both sides of (32) tend to zero as $y \rightarrow 1$. It therefore will suffice to find a solution of (33). Differentiating again and 
using Lemma 2 (with the roles of $x, y$ interchanged) we see that it will suffice to find a solution $f(x)$ of

$$
c_{18}(n) \int_{y / 2}^{1 / 2} f(x) y\left(x^{2}-y^{2} / 4\right)^{(n-3) / 2} d x=l^{\prime \prime}(y) \quad(0<y<1),
$$

or, what is the same, of

$$
\int_{y / 2}^{1 / 2} f(x)\left(x^{2}-y^{2} / 4\right)^{(n-3) / 2} d x=m(y) \quad(0<y<1)
$$

with

$$
m(y)=c_{19}(n) y^{-1} l^{\prime \prime}(y)
$$

7. Solution of the integral equation. Suppose now that $n=2$. Then by Lemma 3 ,

$$
\begin{aligned}
& m(y)=c_{20} y^{-1} \arccos y, \\
& m^{\prime}(y)=-c_{20}\left(y^{-2} \arccos y+y^{-1}\left(1-y^{2}\right)^{-1 / 2}\right) .
\end{aligned}
$$

in $0<y<1$.

The substitution $x^{2}=X, y^{2} / 4=Y$ transforms (35) into an integral equation of Abel type which can be solved in a routine way [2, Chapter 1]. One finds the solution

$$
f(x)=-4 \pi^{-1} \int_{2 x}^{1} x\left(t^{2}-4 x^{2}\right)^{-1 / 2} m^{\prime}(t) d t \quad(0<x<1 / 2) .
$$

Indeed, if we substitute this expression for $f(x)$ into the left hand side of (35), we obtain

$$
-4 \pi^{-1} \int_{y / 2}^{1 / 2} d x\left(x^{2}-y^{2} / 4\right)^{-1 / 2} \int_{2 x}^{1} d t x\left(t^{2}-4 x^{2}\right)^{-1 / 2} m^{\prime}(t) .
$$

The functions in this double integral do not change sign, and we may change the order of integration. This gives

$$
-4 \pi^{-1} \int_{y}^{1} d t m^{\prime}(t) \int_{y / 2}^{t / 2} d x x\left(x^{2}-y^{2} / 4\right)^{-1 / 2}\left(t^{2}-4 x^{2}\right)^{-1 / 2} .
$$

The inner integral equals $\pi / 4$ and we obtain

$$
-\int_{y}^{1} m^{\prime}(t) d t=m(y)-m(1)=m(y)
$$

The function $f(x)$ defined by (39) is continuous in $0<x<1 / 2$ and tends to a finite limit as $x \rightarrow 1 / 2$ : We have only to show that

$$
\lim _{x \rightarrow 1 / 2} \int_{2 x}^{1} x\left(t^{2}-4 x^{2}\right)^{-1 / 2}\left(t^{-2} \arccos t+t^{-1}\left(1-t^{2}\right)^{-1 / 2}\right) d t
$$


exists. But this limit equals

$$
\begin{aligned}
\lim _{x \rightarrow 1 / 2} \int_{2 x}^{1} x\left(t^{2}-4 x^{2}\right)^{-1 / 2} t^{-1} & \left(1-t^{2}\right)^{-1 / 2} d t \\
& =\frac{1}{4} \lim _{x \rightarrow 1 / 2} \int_{2 x}^{1}(t-2 x)^{-1 / 2}(1-t)^{-1 / 2} d t \\
& =\frac{1}{4} \lim _{x \rightarrow 1 / 2} \pi .
\end{aligned}
$$

The function $f(x)$ may therefore be extended to a function which is continuous in $0<x \leqq 1 / 2$. Moreover, (39) implies that

$$
f(x) \ll \int_{2 x}^{1} x\left(t^{2}-4 x^{2}\right)^{-1 / 2}\left(t^{-2}+t^{-1}\left(1-t^{2}\right)^{-1 / 2}\right) d t \ll x^{-1}
$$

as $x \rightarrow 0$, and (31) holds. Finally, when $t$ is small, $\left|m^{\prime}(t)\right| \gg t^{-2}$, and when $x$ is small,

$$
f(x) \gg \int_{2 x}^{\sqrt{ } x} x\left(t^{2}-4 x^{2}\right)^{-1 / 2} t^{-2} d t \geqq \int_{2 x}^{\sqrt{ } x} x t^{-3} d t \gg x^{-1},
$$

and therefore (20) holds.

Next, suppose that

$$
n=3 \text {. }
$$

Differentiating both sides of (35) we obtain

whence

$$
-(1 / 2) f(y / 2)=m^{\prime}(y) \quad(0<y<1)
$$

$$
f(x)=-2 m^{\prime}(2 x) \quad(0<x<1 / 2) .
$$

By virtue of Lemma 4, both sides of (35) approach 0 as $y \rightarrow 1$, and hence (40) does in fact give a solution of the integral equation. The function $f(x)$ is positive and continuous in $0<x<1 / 2$, and tends to zero as $x \rightarrow 1 / 2$. This also follows from Lemma 4. Hence $f(x)$ may be extended to a function which is continuous in $0<x \leqq 1 / 2$. Finally, again by Lemma 4 , we have $x^{-2} \ll f(x) \ll x^{2}$ as $x \rightarrow 0$, and hence (21) and (31) hold.

8. Proof of Lemma 3. By virtue of (26) and (29) we have

$$
l(y)=\int_{S} \int_{S} d \sigma_{1} d \sigma_{2} \int_{E^{n}} d v f_{B}\left(\sigma_{1} ; v ; \iota \mid y \sigma_{2}\right) f_{B}\left(\sigma_{1} ; v ; \iota \mid \mathbf{0}\right) .
$$

The inner integral here can easily be evaluated:

$$
\int d \boldsymbol{v} f_{B}(\boldsymbol{u} ; \boldsymbol{v} ; \iota \mid \boldsymbol{x}) f_{B}(\boldsymbol{u} ; \boldsymbol{v} ; \iota \mid \mathbf{0})=\left\langle\left|u_{1}\right|-\left|x_{1}\right|\right\rangle \cdots\left\langle\left|u_{n}\right|-\left|x_{n}\right|\right\rangle
$$

where

$$
\begin{aligned}
\langle\alpha\rangle=\alpha & \text { if } \alpha \geqq \mathrm{C}, \\
=0 & \text { otherwise. }
\end{aligned}
$$


Assume now that $n=2$. We then obtain

$$
\begin{aligned}
l(y) & =(2 / \pi)^{2} \int_{0}^{\pi / 2} \int_{0}^{\pi / 2}\langle\cos \varphi-y \cos \psi\rangle\langle\sin \varphi-y \sin \psi\rangle d \varphi d \psi \\
& =4 \pi^{-2} \int_{0}^{\pi / 2} d \psi \int_{s(\psi, y)}^{c(\psi, y)} d \varphi(\cos \varphi-y \cos \psi)(\sin \varphi-y \sin \psi)
\end{aligned}
$$

with

$$
c(\psi, y)=\arccos (y \cos \psi), \quad s(\psi, y)=\arcsin (y \sin \psi) .
$$

Straightforward but tedious calculations give

$$
\begin{aligned}
l(y)= & 4 \pi^{-2} \int_{0}^{\pi / 2} d \psi\left(\frac{1}{2}+\frac{1}{2} y^{2}-y \cos \psi\left(1-y^{2} \sin ^{2} \psi\right)^{1 / 2}-y \sin \psi\left(1-y^{2} \cos ^{2} \psi\right)^{1 / 2}\right. \\
& \left.+y^{2} \sin \psi \cos \psi(c(\psi, y)-s(\psi, y))\right) \\
= & 2 \pi^{-2}\left(\left(1+2 y^{2}\right) \arccos y-3 y\left(1-y^{2}\right)^{1 / 2}\right),
\end{aligned}
$$

and Lemma 3 follows immediately.

9. Proof of Lemma 4. Let $l_{2}(y)$ be the function computed in the last section, i.e. the function $l(y)$ with $n=2$. Define a function $p(x, y)$ in the quadrant $x \geqq 0$, $y \geqq 0$ by

$$
\begin{aligned}
p(x, y) & =x^{2} l_{2}(y / x) & & \text { if } y<x \\
& =0 & & \text { otherwise. }
\end{aligned}
$$

By Lemma 3, we have for $0 \leqq y \leqq x, 0<x$ the equations

$$
\begin{aligned}
p(x, y) & =2 \pi^{-2}\left(\left(x^{2}+2 y^{2}\right) \arccos (y / x)-3 y\left(x^{2}-y^{2}\right)^{1 / 2}\right), \\
p_{x}(x, y) & =4 \pi^{-2}\left(x \arccos (y / x)-(y / x)\left(x^{2}-y^{2}\right)^{1 / 2}\right), \\
p_{y}(x, y) & =8 \pi^{-2}\left(y \arccos (y / x)-\left(x^{2}-y^{2}\right)^{1 / 2}\right), \\
p_{y y}(x, y) & =8 \pi^{-2} \arccos (y / x),
\end{aligned}
$$

and for $0 \leqq y<x$ we have

$$
p_{y y y}(x, y)=-8 \pi^{-2}\left(x^{2}-y^{2}\right)^{-1 / 2} .
$$

The functions $p, p_{x}, p_{y y}$ are nonnegative and $p_{y}, p_{y y y}$ are nonpositive.

Now suppose that $n=3$. It follows from (41) and (42) that the function $l(y)=l_{3}(y)$ is given by

$$
\begin{aligned}
l(y)=(2 / \pi)^{2} & \int_{0}^{\pi / 2} \int_{0}^{\pi / 2} d \varphi d \psi \sin \varphi \sin \psi\langle\cos \varphi-y \cos \psi\rangle \\
& \int_{0}^{\pi / 2} \int_{0}^{\pi / 2} d \eta d \theta\langle\sin \varphi \cos \eta-y \sin \psi \cos \theta\rangle\langle\sin \varphi \sin \eta-y \sin \psi \sin \theta\rangle .
\end{aligned}
$$


$(2 / \pi)^{2}$ times the double integral over $\eta, \theta$ equals $p(\sin \varphi, y \sin \psi)$, and we obtain

$$
\begin{aligned}
l(y) & =\int_{0}^{\pi / 2} d \psi \int_{s(\psi, y)}^{c(\psi, y)} d \varphi \sin \varphi \sin \psi(\cos \varphi-y \cos \psi) p(\sin \varphi, y \sin \psi) \\
& =\int_{0}^{\pi / 2} d \psi q(\psi, y),
\end{aligned}
$$

say. The function $q(\psi, y)$ is continuous, and $q(\psi, 1)=0$ since $c(\psi, 1)=s(\psi, 1)=\psi$. Hence (i) holds.

Now suppose that $0 \leqq \psi \leqq \pi / 2$ and $0<y<1$. Then by Lemma 2 ,

$q_{y}(\psi, y)=\int_{s(\psi, y)}^{c(\psi, y)} d \varphi \sin \varphi \sin \psi(-\cos \psi p(\sin \varphi, y \sin \psi)$

$$
\left.+(\cos \varphi-y \cos \psi) \sin \psi p_{y}(\sin \varphi, y \sin \psi)\right) \text {. }
$$

Applying Lemma 2 again we obtain $l^{\prime}(y)=\int_{0}^{\pi / 2} d \psi q_{y}(\psi, y)$. The function $q_{y}(\psi, y)$ is continuous and tends uniformly to zero as $y \rightarrow 1$. Hence (ii) holds.

Another application of Lemma 2 yields

$$
\begin{aligned}
q_{y y}(\psi, y)= & \int_{s(\psi, y)}^{c(\psi, y)} d \varphi \sin \varphi \sin \psi\left(-2 \sin \psi \cos \psi p_{y}(\cos \varphi, y \sin \psi)\right. \\
& +\left(-\cos \psi\left(1-y^{2} \cos ^{2} \psi\right)^{-1 / 2}\right)\left(1-y^{2} \cos ^{2} \psi\right)^{1 / 2} \\
= & \cdot\left(-\sin \psi \cos \psi p\left(\left(1-y^{2} \cos ^{2} \psi\right)^{1 / 2}, y \sin \psi\right)\right) \\
&
\end{aligned}
$$

say. Note that the terms $\left(1-y^{2} \cos ^{2} \psi\right)^{1 / 2}$ in $v(\psi, y)$ cancel out. The function $q_{y y}(\psi, y)$ is continuous and bounded in $0 \leqq \psi \leqq \pi / 2,0<y<1$. It tends to zero as $y \rightarrow 1$, uniformly in every fixed closed subinterval of $0<\psi<\pi / 2$. It tends to a function $w(\psi)$ as $y \rightarrow 0$, where $w(\psi)$ is positive in $0<\psi<\pi / 2$, and the convergence is uniform in every closed subinterval of $0<\psi<\pi / 2$. From all this it follows that $l^{\prime \prime}(y)=\int_{0}^{\pi / 2} q_{y y}(\psi, y) d \psi$, and that (iii) is satisfied.

Again suppose that $0<y<1,0 \leqq \psi \leqq \pi / 2$. Again applying Lemma 2 we obtain

$$
\begin{aligned}
u_{y}(\psi, y)= & \int_{s(\psi, y)}^{c(\psi, y)} d \varphi \sin \varphi \sin \psi\left(-3 \sin ^{2} \psi \cos \psi p_{y y}(\sin \varphi, y \sin \psi)\right. \\
& \left.\quad+(\cos \varphi-y \cos \psi) \sin ^{3} \psi p_{y y y}(\sin \varphi, y \sin \psi)\right) \\
& +2 \sin ^{2} \psi \cos \psi p_{y}\left(\left(1-y^{2} \cos ^{2} \psi\right)^{1 / 2}, y \sin \psi\right) .
\end{aligned}
$$

A little care is needed here since $p_{y y y}$ is not bounded. But

$$
\begin{aligned}
\mid(\cos \varphi-y \cos \psi) \sin ^{3} & \psi p_{y y y}(\sin \varphi, y \sin \psi) \mid \\
& \ll \cos \varphi \sin ^{3} \psi(\sin \varphi+y \sin \psi)^{-1 / 2}(\sin \varphi-y \sin \psi)^{-1 / 2} \\
& \ll \psi^{3} \cos \varphi(\sin \varphi+\sin (s(\psi, y)))^{-1 / 2}(\sin \varphi-\sin (s(\psi, y)))^{-1 / 2} \\
& \ll \psi^{3} \cos \varphi s(\psi, y)^{-1 / 2}(\varphi-s(\psi, y))^{-1 / 2}(\cos \varphi)^{-1 / 2} \\
& \ll y^{-1 / 2}(\varphi-s(\psi, y))^{-1 / 2}
\end{aligned}
$$


and condition (22) is satisfied. Our inequalities imply that $\left|u_{y}(\psi, y)\right| \ll y^{-1 / 2}$, uniformly in $\psi$. Next we observe that

$v_{y}(\psi, y)=\sin \psi \cos ^{2} \psi\left(-y \cos ^{2} \psi\left(1-y^{2} \cos ^{2} \psi\right)^{-1 / 2} p_{x}\left(\left(1-y^{2} \cos ^{2} \psi\right)^{1 / 2}, y \sin \psi\right)\right.$ $\left.+\sin \psi p_{y}\left(\left(1-y^{2} \cos ^{2} \psi\right)^{1 / 2}, y \sin \psi\right)\right)$.

It follows that

$$
l^{\prime \prime}(y)=\int_{0}^{\pi / 2}\left(u_{y}(\psi, y)+v_{y}(\psi, y)\right) d \psi
$$

One has $\left|l^{\prime \prime \prime}(y)\right| \ll y^{-1 / 2}$ as $y \rightarrow 0$, and, as is easily seen, $l^{\prime \prime \prime}(y) \rightarrow 0$ as $y \rightarrow 1$. (For example, by (45) we have

$\int_{s(\psi, y)}^{c(\psi, y)} d \varphi \sin \varphi \sin \psi(\cos \varphi-y \cos \psi) \sin ^{3} \psi p_{y y y}(\sin \varphi, y \sin \psi)$

$$
\ll \int_{s(\psi, y)}^{c(\psi, y)} d \varphi y^{-1 / 2}(\varphi-s(\psi, y))^{-1 / 2} \ll y^{-1 / 2}(c(\psi, y)-s(\psi, y))^{1 / 2},
$$

and this tends to 0 as $y \rightarrow 1$.)

\section{REFERENCES}

1. G. H. Hardy, On differentiation and integration under the integral sign, Quart. J. Math. 32 (1901), 66-140.

2. G. Kowalewski, Integralgleichungen, de Gruyter, Berlin, 1930.

3. F. K. Roth, On irregularities of distribution, Mathematika 1 (1954), 73-79.

4. W. M. Schmidt, Metrical theorems on fractional parts of sequences, Trans. Amer. Math. Soc. 110 (1964), 493-518.

UNIVERSITY OF COLORADO,

Boulder, Colorado 